\title{
O PAPEL DO ENFERMEIRO NA ADESÃO AO TRATAMENTO DE PESSOAS COM TRANSTORNO AFETIVO BIPOLAR: O QUE OS REGISTROS DIZEM?1
}

\author{
Beatriz Pedreira ${ }^{2}$;Marcos Hirata Soares ${ }^{3}$; \\ Anaísa Cristina Pinto ${ }^{2}$
}

\begin{abstract}
Trata-se de estudo descritivo-exploratório que descreve o papel do enfermeiro na adesão ao tratamento da pessoa com transtorno afetivo bipolar (TAB), através do levantamento de registros feitos por estudantes e enfermeiras nos prontuários, e aplicação de uma escala em um serviço comunitário de saúde mental. Utilizou-se a metodologia clínicoqualitativa e, como instrumento de coleta, um diário de campo. A escala foi aplicada no primeiro e último contato com cinco sujeitos do interior paranaense, no período de um mês, em 2010. Percebeu-se uma evolução mais significativa na adesão ao tratamento em somente uma das pacientes. Os registros não relatam conduta de enfermagem na maioria das vezes e possuem aspectos da psiquiatria organicista em sua descrição. O enfermeiro deve registrar suas intervenções e resultados adequadamente.
\end{abstract}

Descritores: Enfermagem Psiquiátrica; Registros de Enfermagem; Transtorno Bipolar; Pesquisa Qualitativa.

\footnotetext{
${ }^{1}$ Artigo extraído de monografia de conclusão de curso "Cotidiano e Adesão ao Tratamento de Pessoas Portadoras de Transtorno Afetivo Bipolar: Estudo de Caso" apresentada ao Centro de Ciências da Saúde, Universidade Estadual de Londrina, PR, Brasil.

${ }^{2}$ Alunas do curso de Graduação em Enfermagem, Departamento de Enfermagem, Centro de Ciências da Saúde, Universidade Estadual de Londrina, PR, Brasil. E-mail: Beatriz - bya_1001@hotmail.com, Anaísa - anaisacristina@hotmail.com.

${ }^{3}$ Enfermeiro, Mestre em Enfermagem, Professor Assistente, Departamento de Enfermagem, Centro de Ciências da Saúde, Universidade Estadual de Londrina, PR, Brasil. E-mail: mhirata@uel.br.
} 


\section{The ROLE OF THE NURSE IN THE ADHERENCE TO TREATMENT OF PEOPLE WITH BIPOLAR AFFECTIVE DISORDER: WHAT DO THE RECORDS SAY?}

Descriptive-exploratory study that described the role of the nurse in the adherence to treatment of the person with bipolar affective disorder (BAD), through the survey of records made by students and nurses in their charts, and application of a scale in a community service of mental healthcare. The clinical-qualitative methodology was used, and as collection instrument, a field log. The scale was applied in the first and last contact with five subjects from the country side of Paraná, in a period of one month in 2010. We observed a more significant evolution in adherence to treatment in only one of the patients. The records do not report the conduct of the nursing staff most of the times and they have aspects of organist psychiatry in their description. The nurses must record their interventions and results adequately.

Descriptors: Psychiatric Nursing; Nursing Records; Bipolar Disorder; Qualitative Research.

\section{EL PAPEL DEL ENFERMERO EN LA ADHESIÓN AL TRATAMIENTO DE PERSONAS CON TRASTORNO AFECTIVO BIPOLAR: ¿QUÉ LOS REGISTROS DICEN?}

Se trata de estudio descriptivo-exploratorio que describe el papel del enfermero en la adhesión al tratamiento de la persona con trastorno afectivo bipolar (TAB), a través del levantamiento de registros hechos por estudiantes y enfermeras en los prontuarios, y aplicación de una escala en un servicio comunitario de salud mental. Se utilizó la metodología clínico-cualitativa y, como instrumento de colecta, un diario de campo. La escala fue aplicada en el primero y último contacto con cinco sujetos del interior paranaense, en el período de un mes, en 2010. Se percibió una evolución más significativa en la adhesión al tratamiento en solamente una de los pacientes. Los registros no relatan conducta de enfermería en la mayoría de las veces y poseen aspectos de la psiquiatría organicista en su descripción. El enfermero debe registrar sus intervenciones y resultados adecuadamente.

Descriptores: Enfermería Psiquiatrica; Registros de Enfermería; Trastorno Bipolar; Investigación Cualitativa.

\section{Introdução}

O transtorno afetivo bipolar (TAB) é caracterizado por alterações de humor que se manifestam como episódios depressivos, alternando-se com episódios de euforia (também denominados mania), em diversos graus de intensidade ${ }^{(1)}$. Entre os fatores de riscos em potencial para o desenvolvimento de TAB encontramse os estressores ambientais, transtornos somáticos e de personalidade, história positiva de TAB na família, situação socioeconômica desestruturada etc. ${ }^{(2)}$.

Um dos maiores desafios no tratamento da pessoa com TAB é conseguir promover a adesão ao tratamento. Vários fatores contribuem para a dificuldade de adesão como a falta de informação a respeito do quadro clínico e do tratamento psicofarmacológico e psicossocial, falta de 
apoio familiar e de vínculo terapêutico adequado com os profissionais da saúde mental, entre muitos outros ${ }^{(2-4)}$.

Com relação à interação com o usuário, num estudo sobre satisfação e adesão ao tratamento de TAB, os resultados mostraram que a maior parte dos pacientes não adere ao tratamento medicamentoso por "comportamento não intencional". A maioria dos sujeitos afirma satisfação com a efetividade do medicamento e com as informações recebidas sobre o mesmo; no entanto, foram identificados relatos de efeitos colaterais, dúvidas e falta de motivação para seguir o tratamento ${ }^{(4)}$.

Nesse contexto, o enfermeiro é um membro-chave para colaborar na adesão e melhora da qualidade de vida da pessoa com TAB, uma vez que permanece grande parte do tempo próximo aos pacientes, tendo oportunidades para educação em saúde, apoio emocional e espaço para a prática do cuidado de enfermagem psiquiátrica, que inclui, entre outras intervenções, o relacionamento interpessoal terapêutico, considerado alicerce para as demais intervenções ${ }^{(5)}$.

Sob a perspectiva ético-legal, os registros de enfermagem, assim como a execução do processo de enfermagem, são recursos fundamentais que colaboram na execução de uma assistência de enfermagem de qualidade que, nesse caso, tem como um dos objetivos motivar a adesão ao tratamento, devendo ser pautada em princípios científicos modernos e estimular o envolvimento do profissional na prática de assistência de enfermagem qualificada.

Os estudos sobre registros de enfermagem são, geralmente, realizados nas áreas de Fundamentos de Enfermagem e Enfermagem Médico-Cirúrgica, onde um exemplo é o estudo que foi realizado num hospital escola ${ }^{(6)}$, o qual concluiu que havia necessidade de aprimorar a qualidade dos registros de enfermagem feitos nos prontuários. Ao se realizar uma pesquisa na base de dados LILACS, no mês de novembro-2010, com as palavras "registros de enfermagem" e "enfermagem psiquiátrica", foram encontrados 8 trabalhos apenas, indicando que ainda há poucos estudos sobre esse tema.

Sendo assim, este estudo visou descrever o papel do enfermeiro na adesão ao tratamento da pessoa com TAB, através de um levantamento de registros feitos por estudantes e pela enfermeira, nos prontuários dos respectivos pacientes, e a aplicação de uma escala de adesão ao tratamento do TAB.

\section{Material e Métodos}

Trata-se de estudo qualitativo, tendo como base a metodologia clínico-qualitativa, aplicada costumeiramente em cenários da saúde, e que permite a possibilidade de articulação de um conjunto de técnicas e procedimentos adequados, típicos do método qualitativo de pesquisa, para descrever e compreender as relações de sentidos e significados dos fenômenos humanos, referidos nesse campo. É concebido como um meio científico de conhecer e interpretar as significações de natureza psicológicas e psicossociais, que os indivíduos dão aos fenômenos do campo saúde/doença ${ }^{(7)}$.

\section{Instrumentos de coleta de dados}

Foi usado como instrumento de coleta de dados o diário de campo e, também, uma escala para prever a adesão ao tratamento para o portador de TAB, elaborada e validada $^{(8)}$, sendo aplicada no primeiro e último contato do entrevistador da pesquisa. Tal escala foi construída como forma de realizar um prognóstico da adesão ao tratamento da pessoa com TAB, e denomina-se escala de adesão ao tratamento para transtorno bipolar do humor (ECPAT$\mathrm{TBH})^{(8)}$, possuindo uma versão resumida, com 21 itens e um coeficiente alpha de Cronbach de 0,79.

As opções desses itens possuem pontuação que varia de 0 a 4 , sendo que, quanto menor a pontuação, maior a probabilidade do paciente de não adesão ao tratamento. $\mathrm{O}$ escore total da escala atinge o valor de 72 pontos e o mínimo, zero. $\mathrm{O}$ fator 1 (questões de 1 a 10 e 14) corresponde à proporção de $40,6 \%$ na explicação do fenômeno adesão. Já o fator 2 (questões 15 a 18) apresenta $29,1 \%$ de proporção de explicação, enquanto que o fator 3 (questão 19), corresponde a $12,3 \%$ e, para os fatores 4 (questão 20) e 5 (questão 21), a proporção de explicação é de 10,7 e $10 \%$, respectivamente ${ }^{(8)}$.

No diário de campo, entre os meses de julho e agosto/2010, registrou-se, pelo menos, uma ou mais cópias semanais dos registros feitos por estudantes de enfermagem e por enfermeiras, nos prontuários dos devidos sujeitos, durante o período de um mês de observação, por se considerar esse um prazo suficiente para ser percebida a relação entre adesão e o papel da enfermeira.

\section{Sujeitos e local}

Avaliaram-se cinco sujeitos com diagnóstico de TAB, no intervalo de um mês. Esses foram atendidos no Centro de Atenção Psicossocial (CAPS) nível III, de um município do interior paranaense. Esse CAPS possui serviço de emergência psiquiátrica agregado à sua estrutura, o que possibilita a internação breve no próprio serviço.

Os sujeitos foram selecionados segundo os critérios de aceitação das normas éticas de pesquisas com seres humanos, ou seja: ter diagnóstico de TAB, ter grau de escolaridade suficiente para compreensão das questões, período ou regime de tratamento no CAPS (internação, intensivo, semi-intensivo e não intensivo), não estar sob tutela ou representado por curador e estar num quadro clínico que possibilite comunicação adequada com o entrevistador.

\section{Normas éticas de pesquisa}

Seguindo os critérios acima, foi solicitado aos profissionais de nível superior que indicassem os nomes de prováveis sujeitos de pesquisa. $\mathrm{O}$ projeto de pesquisa foi aprovado pelo Comitê de Ética em Pesquisa Envolvendo Seres Humanos da Universidade Estadual de Londrina, sob Parecer $n^{\circ} 053 / 10$. 


\section{Resultados}

A análise dos dados obtidos pela aplicação da escala (ECPAT-TBH) e pelo acesso aos prontuários das pacientes, no período de um mês, permitiu organizar as informações coletadas em 3 figuras, sendo as características comuns nos histórico dos pacientes, os escores da escala aplicada no primeiro e último dia de contato e o conteúdo das anotações feitas por estudantes de enfermagem e enfermeiras. Os nomes dos pacientes foram representados por suas iniciais e foram atribuídas letras para os estudantes e enfermeiras. Todas as entrevistadas são do sexo feminino (100\%) com idades entre 21 e 40 anos. As cinco pacientes estão separadas, divorciadas ou solteiras e cursaram somente o ensino fundamental.

\begin{tabular}{|l|l|}
\hline \multicolumn{1}{|c|}{ Sujeitos } & \multicolumn{1}{c|}{ Histórico resumido do sujeito } \\
\hline SEJ & $\begin{array}{l}\text { Infância: dificuldade na escola, uso de anticonvulsivantes, violência intradomiciliar, comportamento agressivo, uso de drogas, } \\
\text { episódios psicóticos, promiscuidade sexual }\end{array}$ \\
\hline RABO & $\begin{array}{l}\text { Infância e adolescência: agitação psicomotora, pensamentos acelerados, alucinações auditivas, ideias paranoicas e persecutórias, } \\
\text { uso de álcool e drogas, pensamento confuso, levando-a a abandonar estudos. Idade adulta: tentativas de suicídio, comportamento } \\
\text { agressivo. Atualmente, mesmo com tratamento, relata problemas familiares, levando-a a baixa adesão ao medicamento }\end{array}$ \\
\hline FALP & $\begin{array}{l}\text { Infância e adolescência: dificuldades na escola. Psicose puerperal, ideias suicidas. Idade adulta: pensamentos e ideias suicidas, } \\
\text { várias internações, tratamento medicamentoso intolerável }\end{array}$ \\
\hline PCM & Infância e adolescência: relato de alucinações auditivas, comportamento social inadequado \\
\hline LP & $\begin{array}{l}\text { Adolescência e idade adulta: tentativas de suicídio, comportamento agressivo, alucinações auditivas, problemas de sono, fobia } \\
\text { social, memória remota prejudicada }\end{array}$ \\
\hline
\end{tabular}

Figura 1 - Histórico resumido dos sujeitos de pesquisa. Paraná, Brasil, 2010

\begin{tabular}{llcc}
\hline Sujeito & \multicolumn{1}{c}{ Regime } & Primeiro contato & Último contato \\
\hline SEJ & Internação breve & $36(19-07)$ & $49(20-08-2010)$ \\
RABO & Intensivo & $53(21-07)$ & $55(24-08-2010)$ \\
FALP & Intensivo & $60(22-07)$ & $64(25-08-2010)$ \\
PCM & Semi-intensivo & $61(21-07)$ & $63(23-08-2010)$ \\
LP & Semi-intensivo & $56(21-07)$ & $59(23-08-2010)$ \\
\hline
\end{tabular}

Figura 2 - Regime de tratamento e resultados da aplicação da Escala ECPAT-TBH. Paraná, Brasil, 2010
$\mathrm{Na}$ Figura 3, devido à impossibilidade de apresentação de todas as anotações, por serem muito extensas, será colocado, no primeiro sujeito, um exemplo de anotação, para que se tome essa como parâmetro de comparação, quando forem feitas as discussões, e para que o leitor entenda qual o sentido dado, ao se atribuir uma característica à evolução de enfermagem como deficiente e/ou somente a descrição do exame de estado mental (EEM) adequado.

\begin{tabular}{|c|c|c|}
\hline Sujeitos & EVOLUÇÃO DE ESTUDANTES DE ENFERMAGEM & EVOLUÇÃO DA ENFERMEIRA \\
\hline 1)SEJ. & $\begin{array}{l}\text { 19/07: "Autocuidado prejudicado - trajando roupas extravagantes, com } \\
\text { libido alterada, consciente, queixando-se de dormir demasiadamente } \\
\text { e acordar tarde por isso, com bom padrão de atenção e compreensão, } \\
\text { parcialmente desorientada em tempo e orientada em espaço, usando } \\
\text { linguagem adequada, porém logorreica, apresentando labilidade de } \\
\text { afeto e humor instável, com memória remota presente e memória } \\
\text { para fatos recentes prejudicada, pensamentos acelerados, com juízo } \\
\text { crítico prejudicado, apresentando delírios persecutórios, alucinações } \\
\text { e ilusões. Já teve ideação suicida, mas não chegou a executar, e no } \\
\text { momento não tem mais”. } 21-07,26-07: \text { “(..)roupas extravagantes, } \\
\text { maquiagens fortes”. Estagiária de enfermagem W. } \\
\text { 02/08: “(...)vestimentas mais adequadas. 09/08: “(...)roupas e } \\
\text { maquiagens extravagantes". } \\
\text { 28/07: “(...)trabalhada a importância de aderir ao tratamento”. Estagiária } \\
\text { de Enfermagem Q. } \\
\text { 03/08: "(...)queixosa”. Outras anotações: 29-07, 02-08,06-08, 13-08. }\end{array}$ & $\begin{array}{l}\text { 31/07: "Ansiosa pela visita do filho, verborreica, irritada, queixosa da } \\
\text { família e nega alucinações. Ligou hoje para a mãe solicitando trazer } \\
\text { o filho para visitá-la. Enf.x". } \\
\text { "À tarde, mais irritada, ansiosa, fazendo ameaças de fugir do } \\
\text { serviço. Conversando com ela, verborreica, se queixando de } \\
\text { obstipação intestinal. Medicada com haldol e fenergan } 1 \text { ampola } \\
\text { cada (IM). Sob cuidados de enfermagem. Com delírios de } \\
\text { perseguição (diz que estão com inveja dela). Enf.x". "Apresentou } \\
\text { períodos de agitação psicomotora. Com melhora espontânea após. } \\
\text { Enf.x". } \\
\text { 06/08: “(...)Conversando com ela, que retornou". } \\
\text { 08/08: "(..)Feitas orientações para usar medicação adequadamente } \\
\text { e retornar, pois está hospedada no CAPS III. Enf.x". } \\
\text { "Mantendo quadro maníaco, sem parada fixa, fala pastosa, } \\
\text { cambaleante, com períodos de irritabilidade e hostilidade, porém } \\
\text { orientada em tempo e espaço, nega alucinações, porém ainda } \\
\text { com delírios persecutórios. Fez cartãozinho para entregar ao pai e } \\
\text { cunhado amanhã (dia dos pais). Enf.x". }\end{array}$ \\
\hline 2) RABO & $\begin{array}{l}\text { Anotações: } 21-07,28-07,04-08,11-08,18-08: \text { As anotações aqui } \\
\text { seguem o mesmo modelo descrito no primeiro sujeito, ou seja, aquele } \\
\text { que apresenta dados apenas do EEM e não de intervenções que tenha } \\
\text { realizado. Estagiária de enfermagem K. } \\
\text { 11-08: “(...)poliqueixosa em relação ao apoio da família. Estagiária de } \\
\text { enfermagem K. }\end{array}$ & $\begin{array}{l}\text { 23/08: Refere que se sentia angustiada, desesperada, querendo } \\
\text { colocar fim à vida. Tentou pôr fogo na roupa, passar faca no } \\
\text { pescoço. Veio de SAMU ao CAPS III, acompanhada pela mãe } \\
\text { e irmã. Quer se internar na CPL, coerente com o desejo da } \\
\text { família. Dr. Y, plantonista, prescreveu medicação, mas suspendeu } \\
\text { após, devido à insistência da paciente para se internar no CPL. } \\
\text { Encaminho à CPL. Sem vaga feminina no CAPS III. Enf.X. }\end{array}$ \\
\hline
\end{tabular}




\begin{tabular}{|c|c|c|}
\hline Sujeitos & EVOLUÇÃO DE ESTUDANTES DE ENFERMAGEM & EVOLUÇÃO DA ENFERMEIRA \\
\hline 3) FALP & $\begin{array}{l}\text { 22-07: “(...)Cuidado exacerbado, usando muitos acessórios e } \\
\text { maquiagem”. 04-08: "(...)chorosa”.11-08: “(...)usando muitos acessórios } \\
\text { e roupas extravagantes”. 15/08: “(..)Orientada a comer em menor } \\
\text { quantidade e a fazer atividade física regular, após a saída do CAPS”. } \\
\text { Outras anotações: } 15-08 \text { e 18-08 (As anotações aqui seguem o mesmo } \\
\text { modelo descrito no primeiro sujeito, ou seja, aquele que apresenta } \\
\text { dados apenas do EEM e não de intervenções que tenha realizado). } \\
\text { Estagiária de enfermagem W. }\end{array}$ & $\begin{array}{l}\text { 06/08: "Ansiosa, diz que quer se desligar um pouco, dormir. Está } \\
\text { sofrendo, pois está gastando sem ter dinheiro, muito irritada, } \\
\text { com vontade de se jogar embaixo de um trem. Conversei com o } \\
\text { plantonista Dr. Y, o qual prescreveu Nitrazepam } 5 \text { mg VO agora. } \\
\text { Enf. x. } \\
\text { 08/08: "Segundo enfermagem, dormiu após a administração de } \\
\text { haloperidol e prometazina } 1 \text { amp cada, IM, pois estava muito } \\
\text { ansiosa. Hoje ainda está apresentando humor lábil, com períodos } \\
\text { de euforia, falando alto, cantando, queixando-se das normas, } \\
\text { triste, desanimada. Abre portas de saída, foi até a cozinha de } \\
\text { funcionários, fica sempre perto dos funcionários, chamando a } \\
\text { atenção". Enf .x. } \\
\text { 12/08: "(...) Orientada a usar roupas adequadas à estação e não } \\
\text { escandalosas". Enf. h.. }\end{array}$ \\
\hline 4) PCM & $\begin{array}{l}\text { Anotações: } 21-07,28-07,04-08,11-08 . \\
\text { 21/07: "Com bom cuidado pessoal, boa aparência, com discurso } \\
\text { conexo, fala organizada, mantendo atenção. Humor e afetos estáveis, } \\
\text { negando ideação suicida. Sem apresentar delírios, orientada em tempo } \\
\text { e espaço, com memória para fatos recentes e remotos preservada. } \\
\text { Apresentado juízo crítico satisfatório. Nega alucinações e ilusões". } \\
\text { Estagiária de enfermagem Q. } \\
\text { As anotações aqui seguem o mesmo modelo descrito no primeiro } \\
\text { sujeito, ou seja, aquele que apresenta dados apenas do EEM e não de } \\
\text { intervenções que tenha realizado. }\end{array}$ & Sem registros da enfermeira \\
\hline 5) L.P. & $\begin{array}{l}\text { Anotações: } 21-07,28-07,04-08,11-08,18-08 \text {. As anotações aqui } \\
\text { seguem o mesmo modelo descrito no primeiro sujeito, ou seja, aquele } \\
\text { que apresenta dados apenas do EEM e não de intervenções que } \\
\text { tenha realizado. } \\
\text { 21/07: "Cuidado pessoal satisfatório, porém, não muito preocupada } \\
\text { com aparência. Em alguns momentos apresentando dificuldade } \\
\text { em manter a atenção, com fala conexa e discurso organizado, } \\
\text { humor e afeto lábeis. Pensamento acelerado, apresentando delírios } \\
\text { persecutórios. Orientada em espaço e parcialmente orientada em } \\
\text { tempo. Memória para fatos recentes presente, e memória remota } \\
\text { ineficaz. Juízo crítico prejudicado, insone. Apresenta alucinações } \\
\text { visuais (vê sombras) e ilusões (vozes de comando, que dizem para } \\
\text { pôr fim à vida). Refere ideação suicida,com planos de atear fogo ao } \\
\text { corpo". Estagiária de enfermagem k. }\end{array}$ & Sem registros da enfermeira \\
\hline
\end{tabular}

Figura 3 - Evolução de enfermagem feita por estudantes de enfermagem e enfermeiras. Paraná, Brasil, 2010

\section{Discussão}

Adesão ao tratamento é definida como a extensão em que os pacientes seguem as recomendações dos profissionais de saúde. Muitas pessoas em atendimento ambulatorial, ou até mesmo após alta hospitalar, têm dificuldade para aderir ao tratamento. Estudos sugerem que, aproximadamente, $50 \%$ dos pacientes bipolares interrompem o tratamento pelo menos uma vez, enquanto $30 \%$ deles o fazem ao menos duas vezes ${ }^{(9)}$.

Conforme evidenciado na Figura 2, todas as pacientes acompanhadas por um mês apresentaram evolução positiva, mesmo que discreta, em aderir ao tratamento, baseando-se nos resultados da escala aplicada no $1^{\circ}$ dia e comparada ao último dia. A paciente SEJ obteve a evolução de maior destaque, com treze pontos na escala e as demais alcançaram de 2 a 4 pontos. Embora seja necessário estudo com amplitude maior, pode-se levantar a hipótese de que a internação breve feita pelo CAPS tenha sido recurso importante no fortalecimento da adesão ao tratamento, além de prevenir internação involuntária ${ }^{(10)}$.

Embora as escalas possuam um bom papel na avaliação da adesão ao tratamento, relações interpessoais terapêuticas ainda são consideradas os melhores preditores de bom prognóstico. A educação para a doença, a identificação e manejo de comorbidades, bem como o estímulo para mudanças positivas no estilo de vida do paciente e sua família, são importantes papéis exercidos pela equipe multidisciplinar no tratamento de pacientes com transtornos de humor ${ }^{(11)}$.

Pensando no sentido de que um dos objetivos alcançados ao longo do relacionamento interpessoal é a promoção de autoestima ${ }^{(5)}$, um estudo de meta-análise ${ }^{(12)}$, envolvendo o total de 1.838 pacientes, sugeriu que a autoestima de pacientes com TAB, em fase de remissão, pode flutuar no decorrer da remissão dos sintomas: é significativamente menor do que nos grupos-controle, e significativamente maior em comparação aos pacientes com transtorno depressivo maior em fase de remissão dos sintomas. Isso indica fortemente que a promoção do relacionamento interpessoal terapêutico é muito importante como fator de adesão ao tratamento, e o item relacionamento e vínculo compõe exclusivamente 3 itens da escala de adesão ao tratamento do $\mathrm{TAB}^{(8)}$. 
Fatores em comum entre as pacientes de acordo com o histórico levantado

Nota-se, na Figura 1, que nos históricos dos sujeitos, há neles a presença do comportamento suicida. Estima-se que até $50 \%$ dos portadores tentem o suicídio ao menos uma vez em suas vidas e $15 \%$ efetivamente o cometem $^{(13)}$. A tentativa de suicídio foi presente na fala da maioria delas, quando observado o item histórico das pacientes, por exemplo, as pacientes 1, 2, 3 e 5. Um estudo sugere que o transtorno bipolar é o transtorno psiquiátrico com maior risco ao longo da vida para tentativas de suicídio e suicídio completo(14). Já sobre a depressão, outro transtorno do humor, um estudo de revisão bibliográfica sugere a depressão ser presente em $70 \%$ das tentativas de suicídio $^{(15)}$

De acordo com o histórico levantado no Quadro 1, todas sofreram algum tipo de abandono familiar, agressão física dos pais ou ex-cônjuges, antes ou após apresentarem os primeiros sintomas do TAB. No estudo conduzido ${ }^{(8)}$, $95,5 \%$ relataram prejuízo ao longo da vida em relação ao trabalho, 88\% com relação à família, 86,6\% nas relações sociais e $85 \%$ no relacionamento conjugal. Esses dados coincidem com os encontrados no histórico dos sujeitos deste estudo em questão.

Em relação a eventos estressores que desencadearam as recorrências, 53,7\% relataram a existência de tais eventos, sendo relacionados à categoria família, com maior proporção $(47,8 \%)$. Apesar de se desconhecer a base causal do TAB, existe interação complexa entre fatores biológicos, genéticos e psicossociais para tentar explicar o transtorno.

A idade de início do aparecimento dos sintomas predominou de 20 a 29 anos, e, apesar da dificuldade em se obter o diagnostico correto, esse resultado vai ao encontro de um autor ${ }^{(16)}$, que afirma que o TAB frequentemente acomete os indivíduos no início de suas vidas profissionais, sendo a idade média de início dos primeiros sintomas de TAB, 20 anos de idade. A mesma literatura discorre também que $69 \%$ dos pacientes são diagnosticados de maneira errônea, sendo a depressão unipolar o diagnostico mais frequente.

Corroborando a prevalência do TAB em idade precoce, um estudo canadense com cerca de 36.984 adolescentes e adultos jovens (15 a 24 anos) apresentou uma prevalência, ao longo da vida, de $3 \%$ e cerca de $56 \%$ deles já estiveram em atendimento médico no último ano; consultando, em média, quatro médicos antes de receber o diagnóstico adequado, e mais de um terço dos pacientes esperam 10 anos ou mais antes de receber o diagnóstico correto, trazendo consequências como: menor probabilidade de que sejam tratados com medicações atualmente recomendadas como primeira linha de tratamento, uso mais frequente de antidepressivos, com seus riscos associados, além de taxas mais altas de suicídio e hospitalização, o que reflete nos desfechos e nos custos ${ }^{(17)}$.
O papel da enfermagem a partir das anotações da enfermeira e estudante de enfermagem

Conforme citado na introdução deste estudo, dos trabalhos encontrados na pesquisa realizada no mês de novembro de 2010, dois estavam relacionados ao tema. Um estudo feito em serviço de emergência psiquiátrica relatou que o conteúdo das anotações pouco tinha contribuído para a avaliação do estado do paciente ${ }^{(18)}$, e uma tese de doutorado ${ }^{(19)}$, que pesquisou a eficácia de um impresso para registro das anotações de enfermagem, concluiu que os alunos que usaram o impresso fornecido pela pesquisadora foram capazes de fornecer todas as informações necessárias, ao passo que os alunos que realizaram as anotações diretamente no prontuário, as fizeram sem fornecer completamente as informações.

Nota-se, pelos registros de enfermagem apresentados na Figura 3 que, na grande maioria das vezes, as informações descrevem o estado mental do paciente com muita propriedade (considerando-se o fato de que são alunos de graduação), quando se observam as anotações feitas por estudantes, por exemplo, a feita sobre o paciente SEJ. Em algumas situações, descrevem condutas tomadas, como orientação medicamentosa e administração de medicamentos, como, por exemplo, sobre os pacientes FALP e SEJ. Já a prática do relacionamento interpessoal como estratégia de cuidado de enfermagem, podendo ser considerada o alicerce para a prática assistencial, não aparece em nenhum dos registros.

As anotações não fornecem um bom parâmetro de avaliação do cuidado prestado, tanto as realizadas pela enfermeira quanto aquelas feitas pelas estudantes, uma vez que há a presença de diversos termos que denotam julgamento moral, como, por exemplo, "roupas extravagantes, maquiagens fortes", feitas para a paciente SEJ. O julgamento moral é algo complexo de ser superado e exige um contínuo olhar para si mesmo, para seus próprios valores éticos e morais, pois é a partir dele que são feitas as avaliações clínicas em saúde mental ${ }^{(20)}$.

Refletindo sobre os achados obtidos nos dois estudos citados aqui $^{(18-19)}$, e ao analisar os registros feitos pelos estudantes na Figura 3, percebe-se que houve melhora na qualidade das informações, quando se analisa a qualidade da descrição do exame de estado mental feito pelas alunas, uma fonte de dados fundamental para se traçar o planejamento das intervenções de enfermagem. No entanto, não são descritas as condutas frente às necessidades dos pacientes, na maioria das vezes.

Nota-se um olhar característico da assistência de enfermagem psiquiátrica organicista, em que o papel do enfermeiro se resume a fazer observações rigorosas do estado do paciente, pautadas no saber baseado na psicopatologia, administrar medicamentos para controlar o comportamento, e informar o médico quaisquer problemas surgidos com os pacientes, sem descrever ou considerar o relacionamento interpessoal como principal recurso terapêutico ${ }^{(5,21)}$. 
As anotações feitas pelo enfermeiro são registros importantes que descrevem a qualidade da assistência de enfermagem prestada. Ou seja, em casos de avaliação ou julgamento ético-moral, acerca do tipo de assistência prestada, os registros demonstram como funciona e se articula a prática, servem de documento legal para comprovar imprudência ou negligência, se é voltada apenas ao fornecimento de medicamentos e descrições baseadas na psicopatologia ou se apresenta outras propostas que demonstrem mais envolvimento do profissional para um cuidado mais humanizado ou, ainda, outras diversas possibilidades explicativas.

Considerando-se a importância do conhecimento do processo de comunicação terapêutica em enfermagem e desse como base para a aplicação do processo de enfermagem, de acordo com a Resolução COFEn

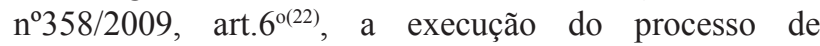
enfermagem deve ser registrada formalmente, envolvendo um resumo dos dados coletados sobre a pessoa, família ou coletividade humana, em um dado momento do processo saúde e doença, como: os diagnósticos de enfermagem acerca das respostas da pessoa, família ou coletividade humana em um dado momento do processo saúde e doença, as ações ou intervenções de enfermagem realizadas face aos diagnósticos de enfermagem identificados e os resultados alcançados como consequência das ações ou intervenções de enfermagem realizadas.

Nas anotações descritas na Figura 3, nota-se o juízo de valor embutido, diminuindo significativamente a qualidade das anotações. Um exemplo são alguns critérios dos registros de enfermagem que, acredita-se, abordaram aspectos fundamentais para descrever os dados relacionados ao paciente no prontuário, quanto à forma, apresentação e especificidade do conteúdo realizado de modo objetivo, sem preconceito, valores, julgamentos ou opinião pessoal. Para esse autor ${ }^{(23)}$, os registros devem evitar generalizações, incluindo termos vagos, como "bom", "regular", "comum", "normal", pois essas descrições tornam-se abertas a múltiplas interpretações, baseadas no ponto de referência do leitor.

As anotações feitas pela enfermeira e estudantes demonstram apenas relatos minuciosos de fatos acontecidos no serviço de saúde, mas não descrevem a participação ativa da enfermagem na grande maioria dos relatos, pois somente em algumas anotações é possível perceber a descrição de atitudes ou intervenções realizadas tanto pelas estagiárias de enfermagem quanto pela própria enfermeira.

Na Figura 3, somente para 2 pacientes, percebese a descrição de intervenções que foram realizadas, quando se lê “(...)trabalhada a importância de aderir ao tratamento(...)" e "(...)Orientada a comer em menor quantidade e a fazer atividade física regular, após a saída do CAPS". Assim, não é possível afirmar se intervenções estão sendo realizadas, mas não anotadas, ou se, realmente, as anotações demonstram com fidedignidade o cenário do cuidado de enfermagem.

Por isso, ainda permanece uma questão a ser esclarecida em estudos futuros. Já que não foi possível compreender se as intervenções são realizadas, porém não são anotadas, ou se, realmente, não são anotadas, na maioria das vezes porque não são realizadas, na maioria das vezes, pois observam-se, na Figura 3, relatos de intervenções feitas com os pacientes, mesmo que limitadas.

\section{Considerações Finais}

A partir da análise feita nos prontuários das pacientes foi possível identificar falhas no registro da assistência de enfermagem prestada ao portador de TAB, permitindo entender que é possível ocorrer omissão dos cuidados, desinteresse em registrar a assistência prestada frente ao quadro clinico apresentado pelas pacientes ou ainda falta de hábito, apenas.

Os resultados deste estudo apontam que os registros efetuados pelas enfermeiras e pelos estudantes não fornecem os dados necessários para se garantir a existência de um processo de enfermagem com qualidade, pois, na sua grande maioria, os registros apresentam o uso de termos de cunho moral e não há diagnósticos, intervenções nem evolução de enfermagem sendo registrados.

Relacionando-se essas reflexões ao papel do enfermeiro na adesão ao tratamento, observou-se que apenas a paciente que permaneceu internada apresentou melhora de adesão diferenciada das demais. Isso suscita ainda várias hipóteses que necessitam maior aprofundamento. Por enquanto, conclui-se que a internação breve configurou-se como diferencial na adesão ao tratamento, uma vez que a paciente com internação breve obteve maior escore em relação às demais pacientes em tratamento no CAPS.

Os registros realizados por estudantes e enfermeiras não consistem em instrumentos sistemáticos de comunicação da e para a qualidade do cuidado de enfermagem. Destaca-se, portanto, a necessidade de esses profissionais se conscientizarem sobre a importância e qualidade desses registros, além de considerar que a sistematização da assistência de enfermagem é tarefa que cabe ao enfermeiro, dando a ele respaldo legal no exercício da profissão.

Ao estar registrado nos prontuários o papel do enfermeiro nos CAPS, haverá maior visibilidade do seu trabalho, o qual visa promover saúde mental no cotidiano do tratamento dessas pessoas, assim como visa permitir aos pacientes expor suas dúvidas, seus anseios, dificuldades, opiniões e experiências relacionadas ao tratamento, buscando melhorar a adesão ao tratamento da pessoa com TAB.

\section{Referencias}

1. Kaplan HI, Sadock BJ, Greeb JA. Compêndio de psiquiatria. 7a.ed. Porto Alegre: Artmed; 2007. 983 p.

2. Gamma JAA, Endras J. Risk Factors for the Bipolar and Depression Spectra. Acta Psychiatr Scand. 2003;108(Suppl):15-9.

3. Miasso AI, Cassiani SHB, Pedrão LJ. Estratégias adotadas por pessoas com transtorno afetivo bipolar e a necessidade de terapêutica medicamentosa. Esc Anna 
Nery. 2007;11(2):240-7.

4. Miasso AI, Monteschi M, Giacchero KG. Bipolar affective disorder: medication adherence and satisfaction with treatment and guidance by the health team in a mental health service. Rev. Latino-Am. Enfermagem 2009; 17(4):548-56.

5. Stefanelli MC, Fukuda IMK, Arantes EC, organizadoras. Enfermagem psiquiátrica em suas dimensões assistenciais. São Paulo: Manole; 2008. 668 p.

6. Carvalho CJA. Análise dos registros de enfermagem em uma unidade cirúrgica de um hospital escola. [Tese de Doutorado]. Ribeirão Preto (SP): Escola de Enfermagem de Ribeirão Preto da Universidade de São Paulo; 2005. 94 p.

7. Turato ER. Tratado da metodologia da pesquisa clinicoqualitativa. 3a.ed. Petrópolis (RJ): Vozes; 2003. 685 p.

8. March R. Escala clínica para prever a adesão ao tratamento: transtorno bipolar do humor. [Tese de Doutorado]. Campinas (SP): Centro de Ciências da VidaPontifícia Universidade Católica; 2008. 240 p.

9. Lingam R, Scott J. Treatment Non-adherence in Affective Disorders. Acta Psychiatr Scand. 2002;105:16472.

10 Ministério da Saúde (BR). Saúde mental no SUS: os centros de atenção psicossocial. Brasília: Ministério da Saúde; 2004. 86 p.

11. Vieira RM, Santin A, Soares JC. O papel da equipe multidisciplinar no manejo do paciente bipolar. Rev Bras Psiquiatr. 2004;26(supl 3):51-3.

12. Nilsson KK, Jorgensen CR, Craig TKJ, Straarup KN, Licht RW. Self-esteem in remitted bipolar disorder patients: a meta-analysis. Bipolar Disord. 2010;12:585-92. 13. Kapczinski FEA. Tratamento farmacológico do transtorno bipolar: as evidencias de ensaios clínicos randomizados. Rev Psiquiatr Clín. 2005;32(supl 1):34-8.

14. Abreu LND, Lafer B, Baca-Garcia E, Oquendo MA. Suicidal ideation and suicide attempts in bipolar disorder type I: an update for the clinician. Rev Bras Psiquiatr. 2009; 31(3):271-80.

15. Chachamovich E, Stefanello S, Botega N, Turecki G. Which are the recent clinical findings regarding the association between depression and suicide? Rev Bras Psiquiatr. 2009; 31(Supl 1):18-25.

16. Costa AMN. Transtorno afetivo bipolar: carga da doença e custos relacionados, Rev Psiquiatr Clín. 2008;35(3):104-10.

17. Kozloff N, Cheung AH, Schaffer A, Cairney J, Dewa CS, Veldhuizen S, et al. Bipolar disorder among adolescents and young adults: Results from an epidemiological sample. J Affect Disord. 2010;125(1):350-4.

18. Galera SF, Capelari RT, Barros S. Estudo das anotações de enfermagem em uma emergência psiquiátrica. Rev Paul Enferm. 1991;10(1):3-12.

19. Forcella HT. Estudo de impressos de anotações de enfermagem psiquiátrica. [Tese de Doutorado]. São Paulo (SP): Escola de Enfermagem de São Paulo da Universidade de São Paulo; 1989. 157 p.

20. Lauder W, Davidson G, Anderson A, Barclay A. Selfneglect: the role of judgements and applied ethics. Nurs Stand. 2005;19(18):45-51.

21. Maftum MA, Alencastre MB. A prática e o ensino de enfermagem em saúde mental e psiquiátrica no Brasil: questões para reflexões. Cogitare Enferm. 2002;7(1):61-7. 22. Conselho Federal de Enfermagem (BR). Resolução COFEN-358/2009. Dispõe sobre a Sistematização da Assistência de Enfermagem e a implementação do Processo de Enfermagem em ambientes, públicos ou privados, em que ocorre o cuidado profissional de Enfermagem, e dá outras providências. [acesso 27 nov 2010]. Disponível em: www.portalcofen.gov.br.

23. Ochoa-Vigo K, Pace AE, Santos CB. Análise retrospectiva dos registros de enfermagem de uma unidade especializada. Rev. Latino-Am. Enfermagem. 2003;11(2):184-91.

\section{Como citar este artigo:}

Pedreira B, Soares MH, Pinto AC. O papel do enfermeiro na adesão ao tratamento de pessoas com transtorno afetivo bipolar: o que os registros dizem?. SMAD, Rev. Eletrônica Saúde Mental Álcool Drog. (Ed. port.). jan.-abr. 2012 [acesso: ];8(1):17-24. Disponível em: 